\title{
The Effect of Korean Red Ginseng on Symptoms and Quality of Life in Chronic Tinnitus: A Randomized, Open-Label Pilot Study
}

\author{
Tae Su Kim¹, Hwan Seo Lee ${ }^{2}$, and Jong Woo Chung ${ }^{2}$ \\ ${ }^{1}$ Department of Otolaryngology, Kangwon National University School of Medicine, Chuncheon, \\ ${ }^{2}$ Department of Otolaryngology, Asan Medical Center, University of Ulsan College of Medicine, Seoul, Korea
}

Received April 28, 2015

Revised July 22,2015

Accepted July 31, 2015
Background and Objectives: The major mechanism of inner ear cell damage is the production of reactive oxygen species (ROS). Korean red ginseng (KRG) has an anti-ROS effect; thus we hypothesized that KRG may be of use for the treatment of chronic idiopathic tinnitus. The aim of the study is to investigate clinical outcomes and health-related quality of life (QoL) in chronic tinnitus patients after taking KRG. Subjects and Methods: This study was an open-label randomized controlled trial. Sixty-one patients with chronic tinnitus were enrolled and randomized to three groups. The control group was treated for 4 weeks with $160 \mathrm{mg} /$ day Ginkgo biloba extract, and two other groups receiving 1500 mg/day or 3000 mg/day KRG for 4 weeks. Clinical assessments were performed using the tinnitus handicap inventory (THI), Visual Analogue Scale (VAS) and QoL was assessed by Short Form-36 Health Survey (SF-36) questionnaire. Results: Fifty-nine patients completed the planned protocol. Significant improvements were observed between initial and post-treatment THI scores in patients receiving 3000 mg/day KRG. There was no statistically significant difference between initial and post-treatment VAS scores in all groups. Treatment with $3000 \mathrm{mg} /$ day KRG for 4 weeks significantly improved role emotional and mental health scores in the SF-36 survey. Conclusions: These results suggest that KRG may improve tinnitus symptoms and mental wellbeing in chronic tinnitus patients.

J Audiol Otol 2015;19(2):85-90

Tel $+82-2-3010-3718$

Fax +82-2-489-2773

E-mail jwchung@amc.seoul.kr

KEY WORDS: Tinnitus · Korean red ginseng · Quality of life.

\section{Introduction}

Tinnitus is generally defined as a perception of sound in the absence of an external acoustic stimulus. It is a common medical complaint that has multiple causes. It has a prevalence of roughly $12-15 \%$ in the adult population [1]. A variety of pharmacological agents have been proposed to reduce tinnitus symptoms, but at present no specific therapy has obtained satisfactory results in all patients. Oxidative stress is suspected to cause idiopathic tinnitus [2,3]. Oral antioxidant therapy in patients with idiopathic tinnitus reduces subjective

This is an Open Access article distributed under the terms of the Creative Commons Attribution Non-Commercial License (http://creativecommons. org/licenses/by-nc/3.0/) which permits unrestricted non-commercial use, distribution, and reproduction in any medium, provided the original work is properly cited. discomfort and tinnitus intensity [3]. Thus, oral antioxidant therapy has been suggested as an additional approach for the treatment of chronic tinnitus.

Korean red ginseng (KRG), an ingredient in traditional oriental medicine for 2000 years, has various beneficial effects on the human body. Pretreatment with ginseng extract significantly attenuates hydrogen peroxide-induced oxidative stress and apoptosis in human SK-N-SH neuroblastoma cells [4]. Ginseng extracts also inhibit reactive oxygen species (ROS) generation, and expression of caspase-3 and poly-ADP-ribose polymerase in cisplatin-induced apoptosis [5]. Furthermore, KRG extracts play both an anti-apoptotic and anti-oxidative role in cisplatin-induced ototoxicity in an auditory cell line $[5,6]$.

A common cause of tinnitus is inner ear cell damage. The major mechanism of inner ear cell damage is the production 
of ROS. KRG has an anti-ROS effect; thus we hypothesized that KRG may be of use for the treatment of chronic idiopathic tinnitus. In addition to clinical measurements like tinnitus handicap inventory (THI) and Visual Analogue Scale (VAS), measurement of health-related quality of life (QoL) also provides an important global assessment of tinnitus patients. The Short Form-36 Health Survey (SF-36) questionnaire has been used in many studies measuring QoL of tinnitus patients $[2,7]$. In this study, we investigated the clinical outcomes and QoL on chronic tinnitus patients after taking KRG. This study was done prospectively using an open-label randomized controlled trial. The control treatment used was Ginkgo biloba, since Ginkgo biloba is widely used as an effective tinnitus treatment $[8,9]$.

\section{Subjects and Methods}

\section{Study design}

The study protocol was approved by the Institutional Review Board of Asan Medical Center. This study was an openlabel randomized controlled trial. Patient consent was obtained before treatment. We enrolled adult patients ( $\geq 20$ years old) with unilateral or bilateral tinnitus that was sustained over 6 months. Patients with a clinical history of psychiatric or neurologic disease, or patients who were taking other drugs during the experiment, were excluded. Exclusion criteria are shown in Table 1. At the first visit, clinical history was evaluated and a physical examination was performed on each patient who had chronic tinnitus. And at the first and last visits, all patients underwent laboratory checks $(\mathrm{CBC}$, chemical and electrolyte), audiological evaluation (pure tone audiometry, speech audiometry, tympanometry, and an auditory brainstem evoked response test), tinnitus evaluation THI and VAS, and SF-36 questionnaire. Mean hearing level was calculated as the pure tone average of $0.5,1,2$, and $4 \mathrm{kHz}$. To exclude neuro-otological problems, computerized tomography scans and/or magnetic resonance imaging was performed.

All patients were randomized into three groups. We planned to have at least twenty patients in each group. The control group was treated for 4 weeks with $160 \mathrm{mg}$ /day Ginkgo biloba extract (Ginexin ${ }^{\circledR}$, SK Chemicals, Seoul, Korea). We administered Ginexin ${ }^{\circledR} 1$ tablet $(80 \mathrm{mg})$ two times in a day to control group for 4 weeks. KRG-medicated patients were randomly placed into two groups, one treated with $1500 \mathrm{mg} /$ day KRG for 4 weeks, and the other treated with $3000 \mathrm{mg}$ /day KRG for 4 weeks. Patients were allocated to the study groups by simple randomization. A computer-generated randomization list was drawn up by the trial coordinator and given to the trial pharmacist. The trial pharmacist then prepared the trial packs according to the randomization sequence. The trial packs consisted of one out of $160 \mathrm{mg} /$ day Ginexin ${ }^{\circledR}, 1500 \mathrm{mg} /$ day and $3000 \mathrm{mg} /$ day KRG for 4 weeks. The trial packs were given to the patients who were unaware of their contents. After informed written consent, the trial packs was opened by the recruiting physician in the presence of the patient. Although patients and physicians were not blinded to the study arms, the statistical analysis was performed in a blinded manner. Capsules of KRG powder were produced by the Korean

Table 1. Exclusion criteria for study enrollment

\begin{tabular}{l} 
Exclusion criteria \\
\hline Middle, inner, retrocochlear ear disease \\
Psychiatric disorders including schizophrenia and depression \\
Neurologic diseases including stroke, transient ischemic attack, \\
Parkinson disease \\
Chronic renal and liver disease \\
Other life threatening diseases including cancer andl \\
myocardia infarction
\end{tabular}

\section{Remarks}

Diagnosed by pure tone audiometry, tympanometry, and/or temporal bone computed tomography

Determined from medical history

Determined from medical history

Determined from laboratory tests

Determined from medical history

Table 2. Characteristics of patients who completed the planned protocol

\begin{tabular}{|c|c|c|c|c|c|c|c|}
\hline & \multirow{2}{*}{ Mean age (yrs) } & \multirow{2}{*}{ Sex (male:female) } & \multirow{2}{*}{$\begin{array}{c}\text { Initial mean } \\
\text { hearing level }(\mathrm{dB} H \mathrm{HL})\end{array}$} & \multirow{2}{*}{$\begin{array}{c}\text { Follow-up mean } \\
\text { hearing level }(\mathrm{dB} H \mathrm{HL})\end{array}$} & \multicolumn{3}{|c|}{ Duration of tinnitus } \\
\hline & & & & & $6 \mathrm{mo}-1 \mathrm{yr}$ & $1-2 y r$ & $\geq 2 \mathrm{yr}$ \\
\hline Group A $(n=20)$ & $55.15 \pm 10.13$ & $8: 12$ & $28.6 \pm 13.25$ & $28.1 \pm 14.68$ & $n=3$ & $\mathrm{n}=5$ & $\mathrm{n}=12$ \\
\hline Group B $(n=20)$ & $52.85 \pm 10.88$ & $7: 13$ & $27.3 \pm 12.72$ & $27.9 \pm 14.34$ & $\mathrm{n}=4$ & $n=3$ & $n=13$ \\
\hline Group C $(n=19)$ & $57.47 \pm 9.71$ & $9: 10$ & $26.8 \pm 11.31$ & $27.2 \pm 12.58$ & $n=4$ & $n=4$ & $n=11$ \\
\hline
\end{tabular}

Mean age and mean hearing level were not significantly different between groups by Wilcoxon signed ranks test. Mean hearing level was not significantly different between initial and follow-up by Wilcoxon signed ranks test. Sex distribution and duration of tinnitus were not significantly different between groups by chi-square test. Group A: control group, treated for 4 weeks with 160 mg/day Ginkgo biloba extract (Ginexin ${ }^{\circledR}$ ), Group B: treated for 4 weeks with 1500 mg/day KRG, Group C: treated for 4 weeks with 3000 mg/day KRG. KRG: Korean red ginseng 
Tobacco and Ginseng Corporation. Each capsule contained $82.15 \%$ KRG extract and $17.85 \%$ miscellaneous gelatin materials. A single capsule contained $500 \mathrm{mg} \mathrm{KRG}$. The daily recommendation dose of KRG for adults is $3000 \mathrm{mg}$. Treatment was initiated the day after randomization. After 4 weeks, medication ceased and all patients were reevaluated for THI, VAS, and SF-36 scores.

\section{Clinical and QoL measurements}

The tinnitus handicap was evaluated with 25 -item THI questionnaire. THI is a widely used questionnaire to assess the severity of tinnitus. A Korean adaptation of THI is composed of 25 items in total, with functional (11 items), emotional (9 items), and catastrophic (5 items) subscales [10]. Three response options ( $0=$ none, $2=$ sometimes, $4=$ always $)$ are available for each item, and the total score is calculated by summing all responses. A total possible score of THI ranges from 0 to 100 , and the higher score of THI represents greater handicap from tinnitus. Intensity of tinnitus symptom was assessed by the patients themselves using VAS with values ranging from 0 to 10 , with higher value designating more intensive symptoms. QoL was presented by general health questionnaire SF-36 completed by patients themselves. The questionnaire included 36 questions and it was designed to measure eight major health domains, including: physical function, physical role, body pain, general health, vitality, social function, emotional role, and mental health. Using certain methodology, scores of domain scales with their values ranging between 0 and 100 were obtained, with higher value designating better QoL [11].

\section{Statistical analyses}

Statistical analyses were performed using the Wilcoxon Signed Ranks test to compare differences between initial and post-treatment THI and VAS scores. Initial and post-treatment changes in THI and VAS (subtracting pre-test scores from post-test scores) were analyzed by one-way ANOVA. The initial difference in SF-36 between groups was evaluated by one-way ANOVA. Effects of KRG on QoL were statistically evaluated using the Wilcoxon Signed Ranks test between initial and post-treatment SF-36 scores. Values of $p<0.05$ were considered significant. All analyses were performed using SPSS 18.0 (SPSS Inc., Chicago, IL, USA).

\section{Results}

Of the 80 patients who were eligible for this study, 61 patients agreed and 19 patients refused to participate. During the study, there were no abnormal findings in laboratory checks after medication except one patient who experienced elevated blood glucose levels. The patient was known to have diabetes mellitus and had been assigned to the $3000 \mathrm{mg}$ KRG group. In the 2nd week of medication, elevated blood glucose levels were reported and thus a doctor of internal medicine recommended that KRG treatment be discontinued. However, we found no correlation between KRG treatment and elevated blood glucose levels. Fifty-nine patients finished the study, as one patient in the $1500 \mathrm{mg} \mathrm{KRG}$ group withdrew from the study for no specific reason. Fifty-nine patients took $\geq 80 \%$ of the required dose of medicine. Characteristics of each group are shown in Table 2. Mean age, sex distribution, mean hearing level and duration of tinnitus were not significantly different between groups. There was no different hearing level between initial and follow-up. After 4 weeks of treatment, THI scores tended to improve in all groups. However, only patients treated with $3000 \mathrm{mg} \mathrm{KRG/day} \mathrm{demonstrated} \mathrm{a} \mathrm{statis-}$ tically significant improvement between initial and post-treatment THI scores $(p<0.01)$. No statistically significant differ-

Table 3. Comparison of initial and post-treatment THI scores

\begin{tabular}{|c|c|c|c|c|}
\hline & \multirow{2}{*}{ Mean \pm S.D. } & \multirow{2}{*}{ Sig.* } & \multicolumn{2}{|c|}{ Difference of THI scores } \\
\hline & & & Mean \pm S.D. & Sig. ${ }^{\dagger}$ \\
\hline \multicolumn{5}{|l|}{ Group A THI $(n=20)$} \\
\hline Initial & $40.25 \pm 14.63$ & \multirow{2}{*}{-} & \multirow{2}{*}{$4.05 \pm 2.22$} & \multirow{2}{*}{-} \\
\hline Post-treatment & $36.20 \pm 19.94$ & & & \\
\hline \multicolumn{5}{|l|}{ Group B THI $(n=20)$} \\
\hline Initial & $39.29 \pm 19.34$ & \multirow{2}{*}{-} & \multirow{2}{*}{$4.38 \pm 2.31$} & \multirow{2}{*}{-} \\
\hline Post-treatment & $34.90 \pm 19.18$ & & & \\
\hline \multicolumn{5}{|l|}{ Group C THI $(n=19)$} \\
\hline Initial & $38.40 \pm 21.61$ & \multirow{2}{*}{$<0.05$} & \multirow{2}{*}{$8.05 \pm 2.33$} & \multirow{2}{*}{$<0.05$} \\
\hline Post-treatment & $30.35 \pm 18.57$ & & & \\
\hline
\end{tabular}

*Wilcoxon signed ranks test, †one-way ANOVA. Group A: control group, treated for 4 weeks with 160 mg/day Ginkgo biloba extract $\left(\right.$ Ginexin $\left.{ }^{\circledR}\right)$, Group B: treated for 4 weeks with 1500 mg/day KRG, Group C: treated for 4 weeks with 3000 mg/day KRG. THI: tinnitus handicap inventory, KRG: Korean red ginseng 
ences between initial and post-treatment THI scores were observed in patients treated with $1500 \mathrm{mg}$ /day KRG or 160 $\mathrm{mg}$ /day Ginkgo biloba extract (Table 3). After 4 weeks of treatment, VAS scores tended to improve in all study groups, although this was not statistically significant (Table 4). There were no intergroup differences in initial SF-36 scores (Table 5). No significant differences were observed between initial and post-treatment SF-36 scores for patients treated with 1500 $\mathrm{mg}$ /day KRG or $160 \mathrm{mg} /$ day Ginkgo biloba extract. However, SF-36 role emotional and mental health scores were significantly higher after 4 weeks of treatment with $3000 \mathrm{mg} /$ day KRG (Table 6). In this study, KRG and Ginkgo biloba extract appeared to be generally safe, and no serious adverse effects have been reported.

Table 4. Comparison of initial and post-treatment VAS scores

\begin{tabular}{|c|c|c|c|c|}
\hline & \multirow{2}{*}{ Mean \pm S.D. } & \multirow{2}{*}{ Sig.* } & \multicolumn{2}{|c|}{ Difference of VAS scores } \\
\hline & & & Mean \pm S.D. & Sig. $^{\dagger}$ \\
\hline \multicolumn{5}{|c|}{ Group A VAS $(n=20)$} \\
\hline Initial & $6.15 \pm 1.90$ & \multirow{2}{*}{-} & \multirow{2}{*}{$0.30 \pm 0.18$} & \multirow{2}{*}{-} \\
\hline Post-treatment & $5.85 \pm 1.96$ & & & \\
\hline \multicolumn{5}{|c|}{ Group B VAS $(n=20)$} \\
\hline Initial & $5.76 \pm 1.90$ & \multirow{2}{*}{-} & \multirow{2}{*}{$0.09 \pm 0.05$} & \multirow{2}{*}{-} \\
\hline Post-treatment & $5.67 \pm 1.83$ & & & \\
\hline \multicolumn{5}{|c|}{ Group C VAS $(n=19)$} \\
\hline Initial & $5.85 \pm 1.96$ & \multirow{2}{*}{-} & \multirow{2}{*}{$0.50 \pm 0.35$} & \multirow{2}{*}{-} \\
\hline Post-treatment & $5.35 \pm 1.90$ & & & \\
\hline
\end{tabular}

*Wilcoxon signed ranks test, †one-way ANOVA. Group A: control group, treated for 4 weeks with 160 mg/day Ginkgo biloba extract $\left(\right.$ Ginexin $\left.{ }^{\circledR}\right)$, Group B: treated for 4 weeks with 1500 mg/day KRG, Group C: treated for 4 weeks with 3000 mg/day KRG. KRG: Korean red ginseng, VAS: Visual Analogue Scale

Table 5. Comparison of initial SF-36 scores between the three groups

\begin{tabular}{lcccc}
\hline & \multicolumn{4}{c}{ Initial scores } \\
\cline { 2 - 3 } & Group A $(n=20)$ & Group B $(n=20)$ & Sig.* \\
\hline Physical function & $73.50 \pm 15.77$ & $79.50 \pm 14.87$ & $78.50 \pm 16.91$ & - \\
Role physical & $72.00 \pm 19.85$ & $75.00 \pm 21.79$ & $69.25 \pm 23.79$ & - \\
Body pain & $69.65 \pm 14.89$ & $74.25 \pm 13.17$ & $67.75 \pm 13.97$ & - \\
General health & $53.93 \pm 13.37$ & $56.46 \pm 13.51$ & $47.08 \pm 14.05$ & - \\
Vitality & $53.21 \pm 15.07$ & $49.63 \pm 16.40$ & $50.25 \pm 17.77$ & - \\
Social function & $52.34 \pm 19.35$ & $48.75 \pm 20.6$ & $48.13 \pm 18.86$ & - \\
Role emotional & $74.47 \pm 24.18$ & $68.33 \pm 23.46$ & $71.67 \pm 22.72$ & - \\
Mental health & $71.45 \pm 18.47$ & $75.30 \pm 16.33$ & $70.60 \pm 15.51$ & - \\
\hline
\end{tabular}

Data are presented as mean \pm S.D. *one-way ANOVA. Group A: control group, treated for 4 weeks with $160 \mathrm{mg} / \mathrm{day}$ Ginkgo biloba extract $\left(\right.$ Ginexin $\left.^{\circledR}\right)$, Group B: treated for 4 weeks with 1500 mg/day KRG, Group C: treated for 4 weeks with 3000 mg/day KRG. SF-36: Short Form-36 Health Survey, KRG: Korean red ginseng

Table 6. Effect of KRG on quality of life, as determined by initial and post-treatment SF-36 scores

\begin{tabular}{|c|c|c|c|c|c|c|c|c|c|}
\hline & \multicolumn{3}{|c|}{ Group A $(n=20)$} & \multicolumn{3}{|c|}{ Group B $(n=20)$} & \multicolumn{3}{|c|}{ Group C $(n=19)$} \\
\hline & Initial & Post & Sig. ${ }^{*}$ & Initial & Post & Sig.* & Initial & Post & Sig.* \\
\hline Physical function & $73.50 \pm 15.77$ & $75.43 \pm 15.89$ & - & $79.50 \pm 14.87$ & $78.00 \pm 17.48$ & - & $78.50 \pm 16.91$ & $74.74 \pm 16.42$ & - \\
\hline Role physical & $72.00 \pm 19.85$ & $69.31 \pm 21.87$ & - & $75.00 \pm 21.79$ & $68.00 \pm 24.65$ & - & $69.25 \pm 23.79$ & $67.10 \pm 23.65$ & - \\
\hline Body pain & $69.65 \pm 14.89$ & $72.19 \pm 12.67$ & - & $74.25 \pm 13.17$ & $75.50 \pm 9.87$ & - & $67.75 \pm 13.97$ & $63.95 \pm 12.88$ & - \\
\hline General health & $53.93 \pm 13.37$ & $51.39 \pm 12.78$ & - & $56.46 \pm 13.51$ & $54.80 \pm 14.25$ & - & $47.08 \pm 14.05$ & $50.22 \pm 12.28$ & - \\
\hline Vitality & $53.21 \pm 15.07$ & $56.13 \pm 15.73$ & - & $49.63 \pm 16.40$ & $55.69 \pm 16.79$ & - & $50.25 \pm 17.77$ & $54.28 \pm 16.48$ & - \\
\hline Social function & $52.34 \pm 19.35$ & $55.13 \pm 18.07$ & - & $48.75 \pm 20.6$ & $53.75 \pm 19.11$ & - & $48.13 \pm 18.86$ & $51.45 \pm 18.67$ & - \\
\hline Role emotional & $74.47 \pm 24.18$ & $79.21 \pm 23.17$ & - & $68.33 \pm 23.46$ & $66.67 \pm 24.52$ & - & $71.67 \pm 22.72$ & $80.26 \pm 21.39$ & $<0.05$ \\
\hline Mental health & $71.45 \pm 18.47$ & $74.97 \pm 14.73$ & - & $75.30 \pm 16.33$ & $72.95 \pm 15.2$ & - & $70.60 \pm 15.51$ & $79.37 \pm 15.04$ & $<0.05$ \\
\hline
\end{tabular}

Data are presented as mean \pm S.D. *Wilcoxon signed ranks test. Group A: control group, treated for 4 weeks with 160 mg/day Ginkgo biloba extract (Ginexin ${ }^{\circledR}$ ), Group B: treated for 4 weeks with 1500 mg/day KRG, Group C: treated for 4 weeks with 3000 mg/day KRG. SF-36: Short Form-36 Health Survey, KRG: Korean red ginseng 


\section{Discussion}

Chronic tinnitus is a pervasive condition that affects many people in a variety of situations. At present, no specific therapy for chronic tinnitus is satisfactory for all patients. A wide range of pharmacological interventions have been proposed for the treatment of tinnitus symptoms. There are a number of reports in the literature suggesting that Ginkgo biloba may be effective in the management of tinnitus [8,9]. Thus, we planned an open-label, randomized design with KRG versus Ginkgo biloba.

In the present study, THI scores tended to decrease in all chronic tinnitus patients after KRG and Ginkgo biloba treatment. However, only patients treated with the recommended dose of KRG showed statistically significant improvements in THI scores, and SF-36 role emotional and mental health scores. Ginkgo biloba and low dose KRG treatment had no significant effect on THI, VAS and SF-36 scores.

Ginseng refers to the root of some species of the genus Panax. Among these species, Panax ginseng C.A. Meyer is the main species cultivated in Korea and the most widely used. The active ingredients of ginseng are termed ginsenosides or ginseng saponins $[12,13]$. Red ginseng refers to the type that is dried after steaming, which may have beneficial properties not found in fresh ginseng. KRG has various health-promoting effects in humans, such as reducing the incidence of cancers, protective effects on gastric ulcers, and anti-inflammatory and anti-oxidative effects $[14,15]$. In addition, KRG has protective effects on hearing loss induced by cisplatin, gentamicin, and noise through protection of outer hair cells; pretreatment with ginseng extract significantly attenuated cisplatin-induced increases in ROS and also inhibited expression of caspase-3 and poly-adenosiediphosphate-ribose polymerase, which was associated with cisplatin-induced apoptosis in HEI$\mathrm{OC} 1$ cells $[5,16]$. However, no previous reports have investigated the effects of red ginseng against tinnitus.

Like other chronic conditions, tinnitus may affect cognitive functioning and cause psychological distress, including anxiety and depression [17]. Tinnitus may negatively impact upon health related QoL, thereby affecting emotional distress of patients to interact normally with other people, making social interaction difficult. This can result in a state of chronic stress that may impact daily activities [18]. Our results showed that KRG reduced particularly role emotional and mental health problem. These results mean KRG had significant benefit for people with chronic tinnitus by improving QoL. KRG is effective for improving the secondary issue of subjective symptom and QoL derived from tinnitus rather than having direct effects on the hearing. The exact mechanism related to subjec- tive symptom and QoL is still unknown, but we can presume that the anxiolytic effect of KRG has an important role as a modulator to improve subjective symptom and QoL. Ginsenosides in KRG interact with the ligand-bindings of the GAB$A_{A}$ and $G_{A B A}$ receptors and induce sedative and anxiolytic effects [7]. These effects may attenuate chronic stress related to tinnitus.

This study has a number of limitations. First, the study was an open-label randomized controlled trial. KRG is also a well-known health food in Korea and regularly consumed. As Koreans may associate KRG with positive benefits, this could in turn affect mental health. Second, Gingko biloba was used as a positive control in this study. But gingko biloba showed no positive effect on chronic tinnitus in this study. Ginkgo biloba is widely used to manage tinnitus; however, multiple, large, well-controlled, double-blind, placebo-controlled clinical trials have found that Ginkgo biloba is no more effective than placebo [19]. Third, this study included insufficient number of patients in each groups. In further study, enrollment of large numbers of tinnitus patients is needed.

Notably, both low dose KRG and Ginkgo biloba had no effect on chronic tinnitus. Taken together, whether Gingko biloba was a positive control or not, these results may attenuate placebo effect of KRG based on preference. Our results suggest that treatment with an effective dose of KRG can reduce tinnitus symptoms and thus influence mental well-being. It is likely that symptomatic psychological distress contributes to the substantial impairment of QoL in chronic tinnitus patients.

\section{Conclusion}

Treatment with $3000 \mathrm{mg} /$ day KRG for 4 weeks significantly improves THI scores and QoL in patients with chronic tinnitus. KRG provides relief to tinnitus patients by reducing tinnitus symptoms, thereby preventing the subsequent negative impact on QoL. These results suggest that treatment with an effective dose of KRG may help alleviate tinnitus.

\section{Acknowledgments}

This study was supported by the grant (2011) from the Korean Society of Ginseng funded by Korea Ginseng Corporation. The tablet of KRG used in this study was supplied by Korea Ginseng Corporation.

\section{REFERENCES}

1) Kvestad E, Czajkowski N, Engdahl B, Hoffman HJ, Tambs K. Low heritability of tinnitus: results from the second Nord-Trøndelag health study. Arch Otolaryngol Head Neck Surg 2010;136:178-82.

2) Neri S, Mauceri B, Cilio D, Bordonaro F, Messina A, Malaguarnera $\mathrm{M}$, et al. Tinnitus and oxidative stress in a selected series of elderly patients. Arch Gerontol Geriatr Suppl 2002;8:219-23. 
3) Savastano M, Brescia G, Marioni G. Antioxidant therapy in idiopathic tinnitus: preliminary outcomes. Arch Med Res 2007;38:456-9.

4) Cheng Y, Shen LH, Zhang JT. Anti-amnestic and anti-aging effects of ginsenoside Rg1 and Rb1 and its mechanism of action. Acta Pharmacol Sin 2005;26:143-9.

5) Im GJ, Chang JW, Choi J, Chae SW, Ko EJ, Jung HH. Protective effect of Korean red ginseng extract on cisplatin ototoxicity in HEIOC1 auditory cells. Phytother Res 2010;24:614-21.

6) Keum YS, Park KK, Lee JM, Chun KS, Park JH, Lee SK, et al. Antioxidant and anti-tumor promoting activities of the methanol extract of heat-processed ginseng. Cancer Lett 2000;150:41-8.

7) Park JH, Cha HY, Seo JJ, Hong JT, Han K, Oh KW. Anxiolytic-like effects of ginseng in the elevated plus-maze model: comparison of red ginseng and sun ginseng. Prog Neuropsychopharmacol Biol Psychiatry 2005;29:895-900

8) Coles R. Trial of an extract of Ginkgo biloba (EGB) for tinnitus and hearing loss. Clin Otolaryngol Allied Sci 1988;13:501-2.

9) Meyer B. [Multicenter randomized double-blind drug vs. placebo study of the treatment of tinnitus with Ginkgo biloba extract]. Presse Med 1986;15:1562-4.

10) Kim JH, Lee SY, Kim CH, Lim SL, Shin JN, Chung WH, et al. Reliability and validity of a Korean adaptation of the tinnitus handicap inventory. Korean J Otolaryngol-Head Neck Surg 2002;45:328-34.

11) Ware JE Jr, Sherbourne CD. The MOS 36-item short-form health survey (SF-36). I. Conceptual framework and item selection. Med
Care 1992;30:473-83.

12) Liu CX, Xiao PG. Recent advances on ginseng research in China. J Ethnopharmacol 1992;36:27-38.

13) Baek NI, Kim DS, Lee YH, Park JD, Lee CB, Kim SI. Ginsenoside $\mathrm{Rh}$, a genuine dammarane glycoside from Korean red ginseng. Planta Med 1996;62:86-7.

14) Yun TK, Zheng S, Choi SY, Cai SR, Lee YS, Liu XY, et al. Non-organ-specific preventive effect of long-term administration of Korean red ginseng extract on incidence of human cancers. J Med Food 2010;13:489-94.

15) Hong CE, Lyu SY. Anti-inflammatory and Anti-oxidative Effects of Korean Red Ginseng Extract in Human Keratinocytes. Immune Netw 2011;11:42-9.

16) Choung YH, Kim SW, Tian C, Min JY, Lee HK, Park SN, et al. Korean red ginseng prevents gentamicin-induced hearing loss in rats. Laryngoscope 2011;121:1294-302.

17) Folmer RL, Griest SE, Meikle MB, Martin WH. Tinnitus severity, loudness, and depression. Otolaryngol Head Neck Surg 1999;121:4851.

18) Henry JA, Dennis KC, Schechter MA. General review of tinnitus: prevalence, mechanisms, effects, and management. J Speech Lang Hear Res 2005;48:1204-35.

19) Hilton MP, Zimmermann EF, Hunt WT. Ginkgo biloba for tinnitus. Cochrane Database Syst Rev 2013;3:CD003852. 